\title{
Marketing Strategy Analysis of Xiaomi Phone Based On Social Media
}

\author{
Wang Xiaobin \\ School of Economic and Management \\ Shenyang Aerospace University \\ Shenyang, China
}

\author{
Li Yaofei \\ School of Economic and Management \\ Shenyang Aerospace University \\ Shenyang, China
}

\begin{abstract}
With the development of mobile internet, behavioral style of everyone and all walks of life in the world is constantly changing, especially the development of smart phone. Xiaomi phone creates one after another marketing miracle through social media. This article studies the marketing strategy of Xiaomi phone under the background of the development of social media, points out the advantage of Xiaomi phone's marketing, provides strong reference of social marketing for other enterprises, and makes suggestions for the sales of Xiaomi phone.
\end{abstract}

Keywords- Social media; Xiaomi phone; social marketing; strategy.

\section{INTRODUCTION}

Nowadays, social media is not only the way people connect each other, but also popular sales mode of companies. So, how does Xiaomi who got the first deal of social media online shopping seize business opportunities? How does Xiaomi attract customers by social media? What's the means of attracting customers? This article will analyze the marketing strategy of Xiaomi phone based on social media.

Beijing Xiaomi Technology Company Limited (Xiaomi Phone Company or Xiaomi Company in short) attracts tremendous attention from the industry since the establishment on April 6, 2010. This is not only because of the founder, Lei Jun, an IT celebrity, but also the sales volume came to 7.19 million sets in the year of 2012 alone, which is RMB 12.65 billion including tax, since the first launch on August 16, 2010. The total amount of tax for the country reached RMB 1.9 billion, which is definitely a miracle in China mobile phone industry. In 2014, Xiaomi still maintains the momentum of rapid development. On the basis of the analysis of the features of social media, applying relevant theories comprehensively, the paper analyzes the marketing strategy and its unique feature of Xiaomi phone based on social media, promotion mode and the solution of problems.

\section{ANALYSIS ON THE STATUS QUO OF HANDSET MARKETING}

\section{A. Analysis on the marketing environment of handset}

Gartner, a professional market research company, said in a report released on February, 2013, the global handset sales volume fell $1.7 \%$ year-on-year in 2012 due to the gloomy economy, preference transfer of customers, fierce competition and so on. Gartner pointed out that the amount of mobile phone sales to end users in 2012 is 1.75 billion, which is a fall of $1.7 \%$ comparing with 2011 .

TABLE I. Comparison of global smart phone sales volume in the fourth quarter of 2012

\begin{tabular}{lrrrr} 
Company & $\begin{array}{r}\text { 4Q12 } \\
\text { Units }\end{array}$ & $\begin{array}{r}\text { 4Q12 Market } \\
\text { Share (\%) }\end{array}$ & $\begin{array}{r}\text { 4Q11 } \\
\text { Units }\end{array}$ & $\begin{array}{r}\text { 4Q11 Market } \\
\text { Share (\%) }\end{array}$ \\
Samsung & $106,957.7$ & 22.7 & $93,830.3$ & 19.6 \\
Nokia & $85,054.8$ & 18.0 & $111,699.4$ & 23.4 \\
Apple & $43,457.4$ & 9.2 & $35,456.0$ & 7.4 \\
ZTE & $16,160.6$ & 3.4 & $18,915.1$ & 4.0 \\
LG Electronics & $14,981.3$ & 3.2 & $16,938.3$ & 3.5 \\
Huawei Technologies & $13,679.1$ & 2.9 & $13,966.1$ & 2.9 \\
TCL Communication & $11,097.6$ & 2.4 & $10,695.3$ & 2.2 \\
Lenovo & $8,305.4$ & 1.8 & $5,206.3$ & 1.1 \\
Sony Mobile Communications & $7,946,6$ & 1.7 & 8.935 .7 & 1.9 \\
Motorola & $7,822.2$ & 1.7 & $10,075.3$ & 2.1 \\
Others & $156,613.7$ & 33.2 & $151,985.1$ & 31.8 \\
Total & $472,076.4$ & $\mathbf{1 0 0 . 0}$ & $\mathbf{4 7 7 , 7 0 3 . 0}$ & $\mathbf{1 0 0 . 0}$ \\
\hline
\end{tabular}

Note: The data in the table comes from the statistical report of Gartner

Reviewing the Chinese mobile phone market, the handset sales have slipped slightly in 2012 after the explosive growth of sales in 2011, but still in a higher level. The main reasons are the strong demand for smart phone that worth RMB1000 or so and strong promotion of terminal handset manufacturers to the sales of mid and low-end smart phone.

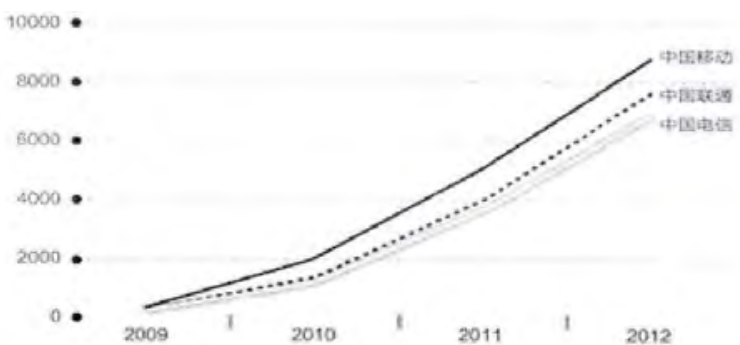

Figure1.Figure of $3 \mathrm{G}$ users quantity in recent 4 years from China Mobile, China Unicom and China Telecom

\section{B. Handset marketing promotion}

In recent years, fierce competition and increasing mobile phone brands feature the mobile phone market. Take the domestic mobile phone as an example, ZTE, Lenovo, Huawei are all the rising brands. However, the traditional handset marketing is relatively monotonous, 
nothing but holiday discount promotion and gifts welfare. The means of handset marketing promotion are diversified in the age of internet with traditional advertising and online advertising; the forms of online advertising have a variety of choices.

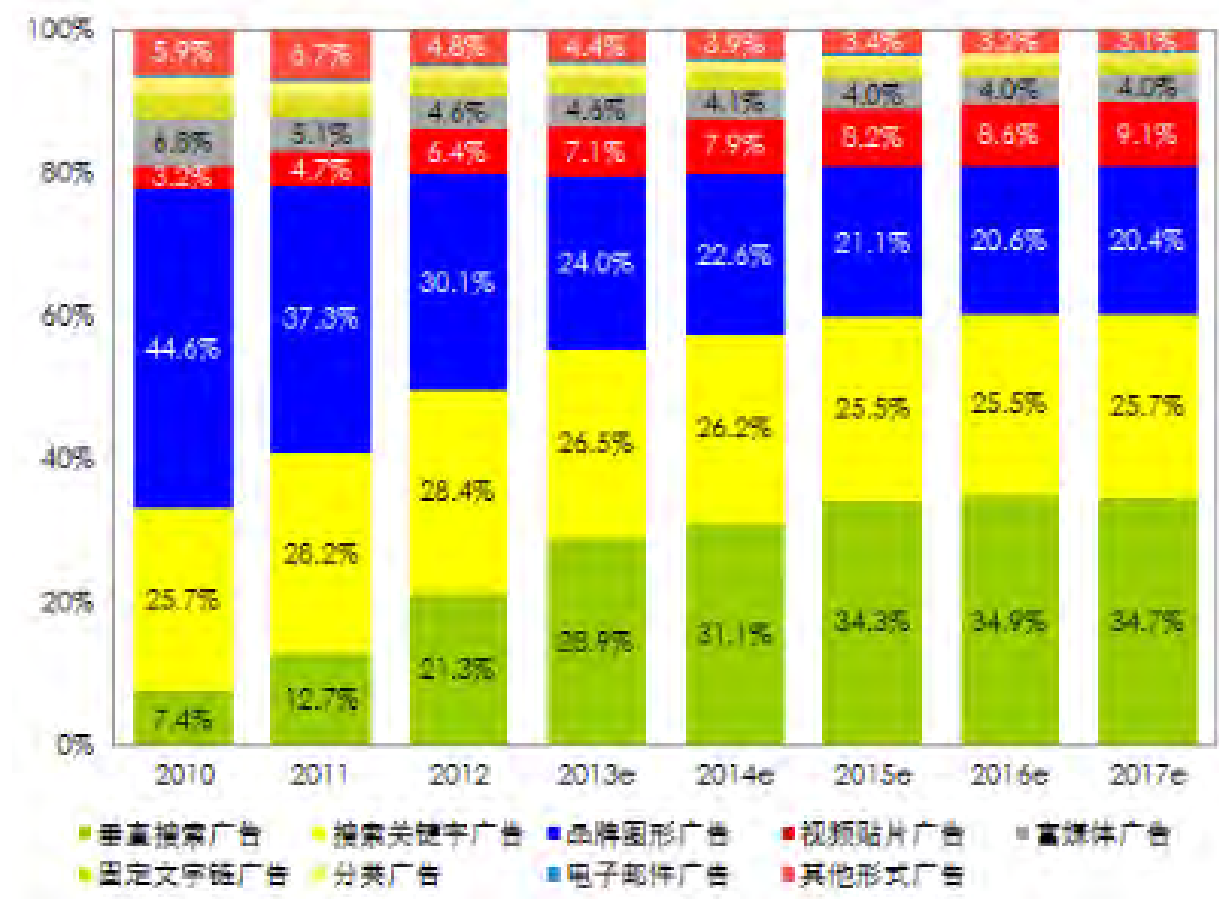

Note: The Figure data roots in enterprise public financial records, industry interviews and model evaluation of IResearch statistical prediction

Figure2. market share and prediction of different online advertising in China from the year 2010 to 2017

\section{Operation model of Xiaomi phone}

The official website of Xiaomi is the main platforms for marketing. The classification on the official website include: Xiaomi phone, accessories, coolplay, 10 points, business hall, service support, MIUI, Mi chat, community, etc. The official website of Xiaomi can basically meet the operating requirements

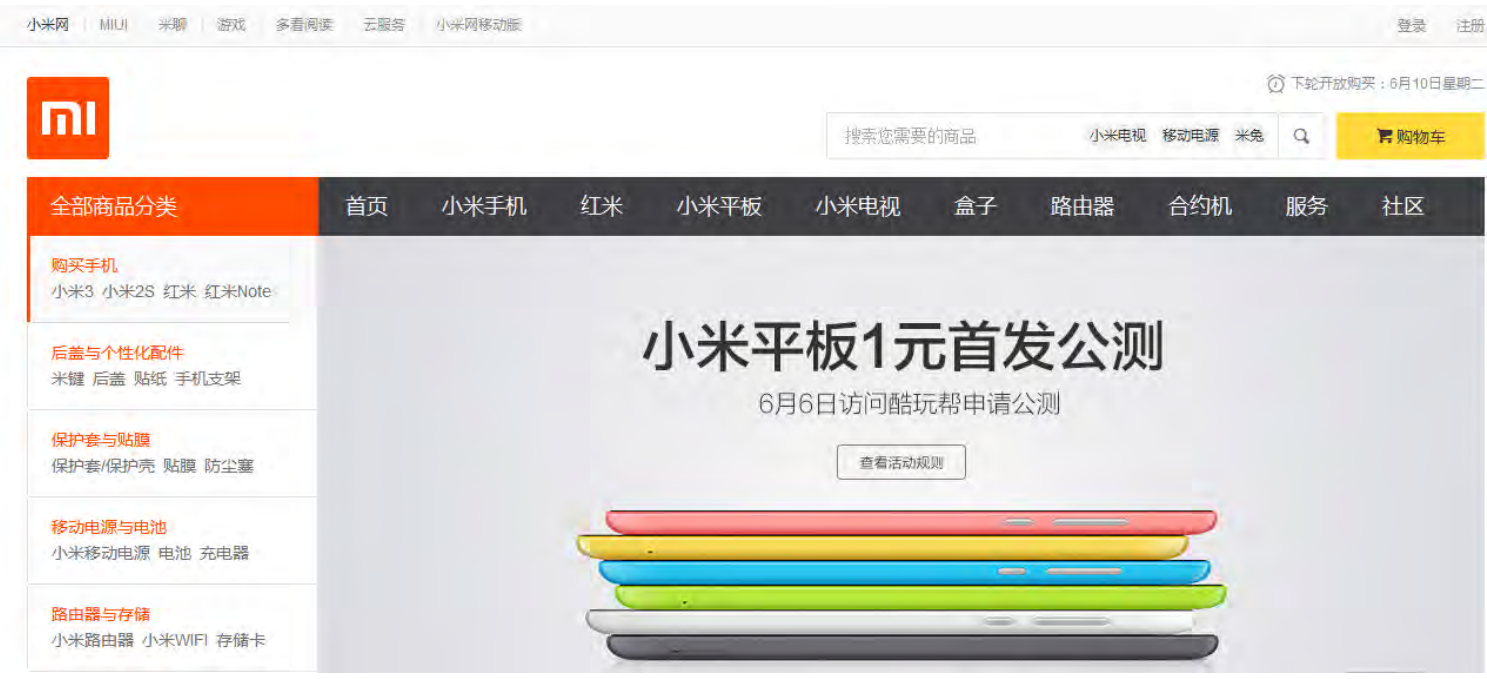

Figure4. Official website home page of Xiaomi phone 
Any new product launch will be first shown on the Xiaomi official website as well as the technical upgrade or patch and different activities. On the official website, you can see the product introduction, business hall, BBS for Xiaomi fans, interactive activities between manufacturers and fans, etc.

Xiaomi adopts the way of online sales, which not only save the cost of market and channel, but also is very fashionable. Vancl provides the logistics and inventory for Xiaomi, and this will definitely save cost for Xiaomi.

\section{SOCIAL MARKETING STRATEGY ANALYSIS ON XIAOMI PHONE}

\section{A. Hunger marketing}

In terms of sales, Xiaomi is a good example of Venture Company. As a venture company, Xiaomi needs to find the balance between alleviating the pressure of Xiaomi fans and output of factories with no mistake at all. Pressure mode plays an important role in this context of "wolves and tigers on both sides".

"Hunger marketing" means commodity provider cut production consciously so as to regulate and control the supply-demand relationship, make short supply illusion, maintain high commodity price and profit margin. The premise of "hunger marketing" is that the brand and quality have enough power, which magnify the value and appeal, lay a foundation for the on-going hot sell, and establish high loyalty of customer group. The hunger marketing of Xiaomi is to fully arouse the attention of consumers through all kinds of strategies of limited amount and limited time, stimulate the buying inclination, and realize the rapid transfer of products from manufacturers to consumers. This marketing strategy makes use of the mind of gaining petty advantages of consumers, and such promotions are all time-limited. If you lose this very rare opportunity, others will grasp, which is the so-called "Less is more".

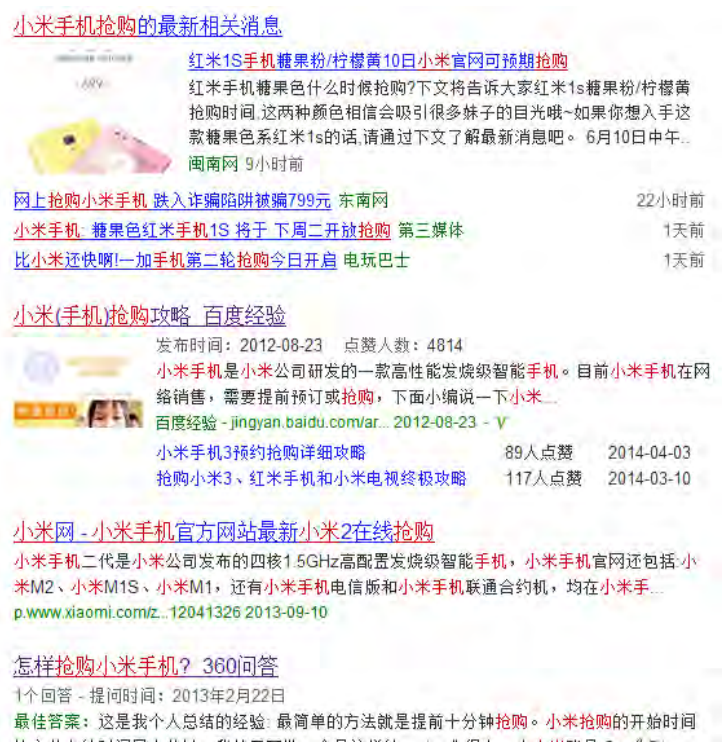

Figure5. Screenshot of keyword search about panic buying of Xiaomi

\section{B. Concept marketing}

Concept marketing is to convey the brand culture through concept conversion and concept spread so as to achieve the goal of recognition and purchasing from consumers. Concept marketing is a kind of leverage, which can launch consumer cognition, release consumption desire, and become popular in the market within just a few months.

With the rapid development of Internet, the number of Weibo users is setting new records constantly. By the end of 2011, the net citizens have reached 500million, which is nearly $300 \%$ higher than 2010. Weibo marketing is a better tool for its low cost, long tail effect and high transmission. Xiaomi phone is actively using Weibo for marketing, which greatly improves the influence on consumers.

\section{Event marketing}

Event marketing refers to improving the popularity and reputation of enterprise and products by means of planning, organizing and utilizing the people or event with news value, social impact, and celebrity charm, attracting the interest and attention of media, social community and consumer, and promoting the means and manner of selling product and service. Due to the widespread audiences and strong abruptness, the effect of information spread can be maximized and optimized in a short time, which will save lots of promotion costs for enterprise, so event marketing becomes more and more popular at home and abroad for public relation and market promotion.

The rumor that Xiaomi handset is stolen has been spreading, and the Xiaomi has no official clarification about the rumor, which arouses verbal battle of Mi fans and Meizu supporters. The appearance of Xiaomi in the sight of cyber covers a layer of "mysterious color" on the Xiaomi phone.

The upcoming news of Xiaomi is often half-block and half-exposed, allowing people to guess. The second- kill of engineering samples came to a conclusion; Mi fans not qualified for the activities are tensing themselves for the next time. At first, the number of limited reservation is 10000 sets from the rumor and has no qualification restriction. The people updating Xiaomi became tumultuous in the BBS. This marketing strategy is just like the way Apple Company does. Apple will make a show of power painstakingly before the new arrival; the media and fans will follow the tracks as half-released messages, and Apple issues the new product under the focus of millions of people. In conclusion, it aims to expand the range of spread. Creating momentum can be a kind of speculation. How to let more e-pals know and join in this activity? Xiaomi will keep high-profile when the release of new phone. By the means of advertorial and BBS promotion, creating anticipation first and then consumers will remember the activity.

\section{Mi fans culture}

What's your first reaction at the mention of Xiaomi phone? Copycat, cheap, or domestic goods? 
The impression of Xiaomi for most people who know little about it is limited to this, but the development of Xiaomi was unexpected. The owners of Xiaomi phone declare themselves as "Mi fans", which is the major force of Xiaomi getting involved in the fashion culture and being popular suddenly. The regularly held "Mi fans festival", rip-roarious BBS, the decorations, accessories, $\mathrm{t}$-shirts, photography, music and micro film derived from Xiaomi compose the content of Xiaomi culture together. The fans culture of Xiaomi phone actually possesses the sample characteristics of public consumer culture nowadays, and shows its significance of meaningful production, pleasure economy and identity politics.

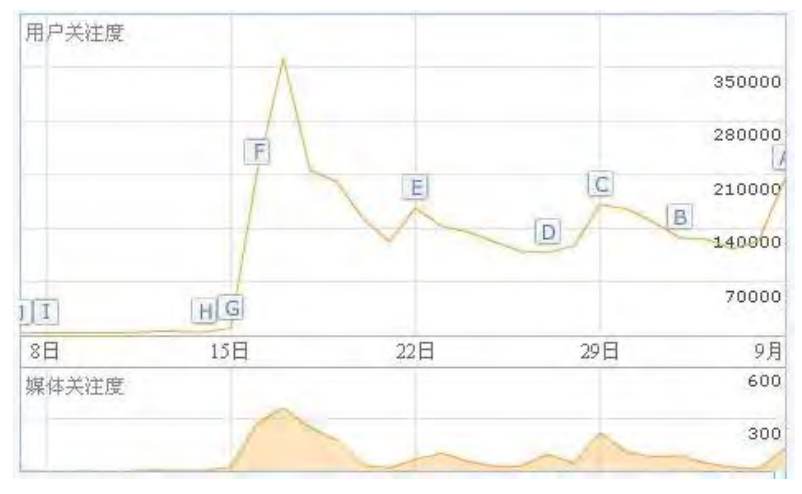

Figure6. Xiaomi handset awareness in the Mi fans festival

For Xiaomi handset, the annual regular Mi fans festival and the launch event of each new style, the dedicated goal is to complete the ceremony spread of Xiaomi culture. The start of Mi fans festival and the interaction between fans and merchant are defined at fixed time, the sense of ceremony in the form of fixed time meets the needs of sense of belonging of fans at the most extent.

\section{COUNTERMEASURES AND SUGGESTIONS TO PROMOTE XIAOMI MARKETING STRATEGIES}

\section{A. Position explicitly, expand market and improve user experience}

The application size of mobile internet is constantly expanding, in view of the online sales mode of Xiaomi phone, it's absolutely possible to market on the mobile terminal, in addition, the transaction size is constantly expanding. The overall transaction scale of online shopping is RMB1,850 billion in 2013, and transaction scale of mobile shopping is RMB167.64 billion

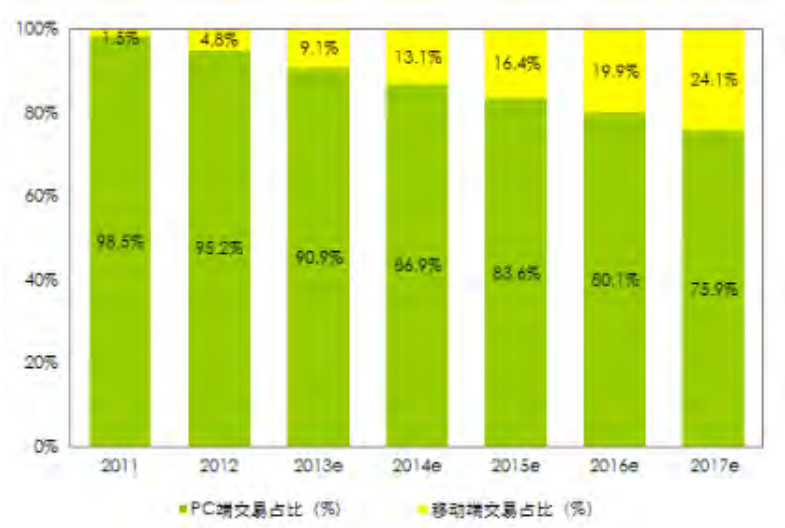

Figure7. Proportion drawing of PC and mobile trading volume of China in 2011-2017

Therefore, Xiaomi phone can design and sell against mobile terminal, for example, Wechat that is in widespread use in handset users. "Dear, did you scan today? Dear, did you shake today?" Wechat now is the most gorgeous elements nowadays. Hence, Wechat brings opportunity for the sales of Xiaomi phone. As we all know, orientation of Wechat is to start Wechat marketing; Xiaomi phone can operate a "personalized magazine brand" according to the Wechat orientation. In short, Xiaomi can provide customized services and a series of convenient services for users paying attention to official Wechat of Xiaomi phone.

In addition, products of many industries are now joining the ranks of free trial; Xiaomi adopts the way of "write down your feelings after use". If you're can't attain ideal experience, you will receive some gifts as feedback or customer service will call back and record the deficiencies and praises, which acquires rich user experience information and then releases on the internet as promotional materials, which is much more persuasive than the promotional materials made up by the copy writer. Thus, Xiaomi positions explicitly and expands market while improves user experience, both of which can also promote each other and bring more comprehensive and optimized development.

\section{B. Enhance the capability of innovation and independent research and development}

As is known to all, Xiaomi has not enough independent property right. The layout of Xiaomi created by Lei Jun is "terminal + content + service". The application of Xiaomi hardware is interconnected; original accessories suppliers include Qualcomm, Sharp, Samsung, TPK, Wintek and Desai, etc. With the support of the top suppliers, Xiaomi shows the shortcomings of not enough independent property right. For now, Xiaomi encounters no big troubles on independent property right, but it will be the threat in the future for sure. So, Xiaomi is bound to strengthen independent $r \& d$ and innovation ability so as to enhance their power.

In this respect, my personal advice is collaborative innovation. Xiaomi may build factories near the colleges and universities in order to attract talents 
contact the institute and promote tripartite cooperation. In this way, the three sides will benefit from each other. I believe that Xiaomi will become the market leader of domestic handset if synergy innovation goes well.

\section{Enhance quality management and ensure the after- sales service}

From the beginning of the sales, Xiaomi has a lot of quality problems, such as rake angle of screen, paint loss, not tight crack closure of back cover, noise when shaking the body which are mostly discussed in the BBS. Therefore, Xiaomi must strengthen the quality management based on the long-term development orientation. Only this can make the quality problems won't be an obstacle to the development of Xiaomi.

Xiaomi promises the after-sales service policy as: users that buy Xiaomi handset will be provided the services of "three guarantees", namely, for repair, replacement or compensation of faulty products. Commitment of Xiaomi Company: products sold (subject to the actual receiving date), according to the detailed rules of "three guarantees" can be returned within 7 days and be replaced according to the detailed rules within 15 days. In addition, Xiaomi provides services including mailing, home delivery, return and replace of products, maintenance, software and hardware, etc. However, the after-sales service of Xiaomi is not satisfactory in reality.

\section{Create meaning in the brand of Xiaomi for sustainable development-}

The development of Xiaomi is like splitting bamboo. It's undeniable that Xiaomi is still a new brand, and popularity still needs to be improved; the brand meaning needs to be shaped. I believe that the name and explanation of LOGO are relatively complicated, somewhat less concise and not suitable for the understanding of the general public. I suggest that the reversed logo of Xiaomi is like the Chinese character “心” less a dot, which means saving worry for customers. Propagate the explanation of "save worries for customers", enhance the interaction with customers and shorten the distance with customer.

\section{CONCLUSION}

Since 2011, Xiaomi phone adopted brand new social media for marketing and created sales miracles one after another, which aroused widespread concern in the industry. In view of this, the article sorts out social marketing theory by studying the domestic and foreign theories, analyzes the handset marketing strategy, social marketing and achievements of Xiaomi phone. What's more, the article evaluates the effect of social marketing of Xiaomi phone, points out existing problems of social marketing of Xiaomi phone and proposes corresponding countermeasure.

Xiaomi phone adopts the way of online sales, which saves cost and is very stylish. However, with the increasingly competitive handset market and after-sales service problems, the paper expects Xiaomi will combine online and offline marketing mode in the future. Under the influence of Xiaomi handset, we experience the power of social media marketing to enterprise. The paper argues that there will be more and more companies attempting social marketing and promoting the development of social marketing.

\section{REFERENCES}

[1] Guo Guoqing. Introduction to marketing[M]. Beijing: China Renmin University Press, 2013:212-229

[2] Clara Shih, Zhang Xiaowei. SNS triggers business and society changes in social networking age[M]. Beijing: Posts and Telecom Press, 2011

[3] Zhang Chunhong. Basis and development cases of social network technology $[\mathrm{M}]$.Beijing: Posts and Telecom Press, 2012.05

[4] Qiu Daoyong. Wechat changes the world[M]. Beijing:Chian Fortune Press, 2013.03

[5] Wang Daen(pen name: Wang Yi), Cao Xueyi (Pen name: Lan Yao ) . Wechat, earn money like this[M].Beijing: China Machine Press, 2013.07

[6] David Meerman Soctt. Real-time Marketing\&PR at microblog era[M]. Beijing: China Machine Press 2012.01

[7] Yan Yan. Microblog changes future@ you can also succeed like this[M]. Taihai Publishing House 2011.07

[8] He Lin. Study on the development strategy of Xiaomi company[D]. Beijing University of Posts and Telecommunications, 2013

[9] Hou Bin. Online and offline integral marketing transmitting strategy study on Motorola handset. [D]. Beijing University of Posts and Telecommunications, 2011

[10] Jin Xing. Course of practical advertising[M] Fudan University Press 2013.09

[11] Liu Linqing, Liu Liu. Advertising outline[M]. Beijing: China Renmin University Press, 2013:107-132

[12] Gao Ming. Advertising Management[M]. Shanghai: Fudan University Press, 2008:89-107

[13] Ding Limin, Sun Dingli. Analysis on the marketing strategy of Xiaomi phone[J]. Hebei Enterprise, 2012,(8)

[14] Li Baomeng, Sun Bo, Yang Longfei. Analysis on the ecommerce operation mode of Xiaomi phone[J]. Ecommerce,2012(9)

[15] Zhang Xuegao. Analysis on the hunger marketing of Xiaomi phone[J] Modern commerce, 2013,(2)

[16] Liu Zhenhua. Marketing philosophy of Xiaomi phone[J]. Modern marketing(Xueyuan edition),2012,(12)

[17] Li Shijie. Xiaomi tops the complain ranking as Improper service [N] Communication information Paper, 2014-3-19(1).

[18] Hu Jun. Background of'processor swap" of Xiaomi3 [N].China Consumers, 2014-1-22(1).

[19] Deng Lunan. Marketing strategy study of Xiaomi phone under the background of social media development[D] East China University of Science and Technology, 2013

[20] Zhang Peng. Study and realization of mobile social network service based on handset address list[D]. Beijing University of Posts and Telecommunications, 2011

[21] Weinberg (United States).Outbreak marketing revolution: social network marketing guide[M], Machinery Industry Press.2010.

[22] David Bartlett(United States).Tell you the focus: Perfect PR[M].Guang dong Economic Press.2011

[23] Dan Skoda Burr(United States).The rise of the personal brand E era[M].Hua zhong University of Science and Technology Press.2011. 